\title{
Training and Support system in the Cloud for improving the situational awareness in Search and Rescue (SAR) operations
}

\author{
Michal Pelka and Karol Majek \\ and Janusz Bedkowski and Pawel Musialik \\ and Andrzej Maslowski \\ Institute of Mathematical Machines \\ ul. Krzywickego 34, 02-078 Warsaw, Poland \\ Email: januszbedkowski@gmail.com
}

Antonio Coelho and Ricardo Goncalves and Ricardo Baptista

DEI, Faculdade de Engenharia, Universidade do Porto / INESC TEC

Rua Dr. Roberto Frias, s/n, Porto, Portugal

E-mail: acoelho@fe.up.pt

Shashank Govindaraj

Space Applications Services NV/SA

Leuvensesteenweg 325, 1932 Zaventem, Belgium

Email: shashank.govindaraj@spaceapplications.com

\author{
Geert de Cubber and Haris Balta \\ Royal Military Academy of Belgium \\ Department of Mechanics, Unmanned Vehicle Centre \\ Avenue de la Renaissance 30, 1000 Brussels, Belgium \\ Email: haris.balta@mil.be \\ geert.de.cubber@rma.ac.be
}

Jose Manuel Sanchez

IntegraSys S.A.

Esquilo 1, 28232 Las Rozas, Spain

Email: jose.sanchez@integrasys-sa.com

\begin{abstract}
In this paper, a Training and Support system for Search and Rescue operations is described. The system is a component of the ICARUS project (http://www.fp7-icarus.eu) which has a goal to develop sensor, robotic and communication technologies for Human Search And Rescue teams. The support system for planning and managing complex SAR operations is implemented as a command and control component that integrates different sources of spatial information, such as maps of the affected area, satellite images and sensor data coming from the unmanned robots, in order to provide a situation snapshot to the rescue team who will make the necessary decisions. Support issues will include planning of frequency resources needed for given areas, prediction of coverage conditions, location of fixed communication relays, etc. The training system is developed for the ICARUS operators controlling UGVs (Unmanned Ground Vehicles), UAVs (Unmanned Aerial Vehicles) and USVs (Unmanned Surface Vehicles) from a unified Remote Control Station (RC2). The Training and Support system is implemented in SaaS model (Software as a Service). Therefore, its functionality is available over the Ethernet. SAR ICARUS teams from different countries can be trained simultaneously on a shared virtual stage. In this paper we will show the multi-robot 3D mapping component (aerial vehicle and ground vehicles). We will demonstrate that these 3D maps can be used for Training purpose. Finally we demonstrate current approach for ICARUS Urban SAR (USAR) and Marine SAR (MSAR) operation training.
\end{abstract}

\section{INTRODUCTION}

The main and most important task of rescue services during a major crisis is to search for human survivors on the incident site. As such endeavour is complex and dangerous, it often leads to loss of lives among the rescuers themselves. Introducing unmanned Search And Rescue (SAR) devices can decrease the scale and frequency of such events, while speeding up the SAR process.

Many research efforts towards the development of unmanned SAR tools have been made(see here for an overview: (Kruijff et al., 2012)). One of these efforts is Neptus, a C3I (Command, Control, Communication and Information) framework, which aims to support coordinated operation of heterogeneous teams, including several types of UVs and human beings (Dias, 2006). Another example, is the German project I-LOV, which establishes a framework for integration of mobile platforms into a victim search mission (Hamp, 2013). Numerous attempts to use robotic systems in crisis situations were made: the 2001 World Trade Center attack (Murphy, 2004), the 2004 earthquake in Mid Niigata, the 2005 USA hurricanes (Murphy, 2008), or the 2011 Japan tsunami. Paper (Liu and Nejat, 2013) and (Hamp, 2013) give a broad overview of the effort done in this area. This research effort stands in contrast to the practical reality in the field, where unmanned SAR tools have great difficulty finding their way to the endusers, due to a number of remaining bottlenecks in the practical applicability of unmanned tools (Doroftei et al., 2012).

This paper focuses on the training and support component of the ICARUS system (Govindaraj et al., 2013). The main goal of ICARUS is to combine robotic components into a common technology platform, capable of increasing the situational awareness of the action in a SAR mission. The system consists of collaborative Unmanned Ground Vehicles (UGVs) and Unmanned Aerial Vehicles (UAVs), operated using novel command and control tools. The data gathered by these unmanned systems is processed, in real-time, by a mobile data centre to provide a high-quality geo-referenced 3D model 
of the environment. Such a model increases the awareness of human crisis managers, can be used in mission planning or can be used for virtual training purpose.

The paper is organized as follows: after an introduction, the architecture of the system is described, followed by brief description of the hardware. Section 4 concentrates on the methodology of data fusion. Section 5 discusses the the results of experiments done with the system. The training tools are presented in section 6 . The paper finalizes with conclusions.

\section{ARCHITECTURE OF THE TRAINING AND SUPPORT SYSTEM}

The Training and Support system is implemented using a SaaS (Software as a Service) model with the capability to perform High Performance Computing in the Cloud (HPC). The HPC approach is a relatively new topic, especially in mobile robotics. We are using it for the task of 3D mapping, which will be described in more details in next sections. HPC is connected with GPU virtualisation technology, allowing many users to have access to computational demanding applications (NVIDIA CUDA parallel computing, virtual training, visualization of 3D cloud of points) in the Cloud. In our opinion, these applications could be used to improve the situational awareness of Search and Rescue operations. We decided to provide needed computation power through a mobile data centre based on NVIDIA GRID server (Supermicro RZ-1240iNVK2 with two VGXK2 cards - in total 4 GPUs with 4GB RAM each) capable of GPU virtualization. For virtualization, we have used the Citrix framework VDI (desktop virtualization) and XenApp (application virtualization - allows GPU sharing between users) infrastructures (figure 1). Published applications can be accessed as SaaS from web browser after installing Citrix Receiver - thin client compatible with all popular operating system for mobile devices (Mac OS X, Linux, Windows, iOS, Android etc.). Current GRID drivers have some limitations in programming framework (CUDA 5.5) and GPU class (CUDA 3.0 CC). Nevertheless, the provided functionalities are sufficient for our applications. Besides CUDA, GRID technology supports building applications with sophisticated rendering (OpenGL 4.4). Thus, in our system it is used for registering, rendering and processing 3D point clouds and for building training tools.

The data-centre can be used in two ways: as a classical data and computation power distribution point, connected to the Ethernet in a safe location, or as a mobile data-center for the robotic teams. In the later scenario the server is placed in a robust carrying case for safety (figure 2. The server is then connected to a router that creates a local network for use by the rescuers. All software on the server can be run on a standard laptop, provided it is with CUDA-compatible GPU.

\section{3D MAPPING HARWARE}

In figure 2 , the examples of ICARUS SAR 3D mapping hardware are shown a) Teodor equipped with a 3D laser scanner based on a rotary SICK LMS 100, b) DrRobot Jaguar 4x4 equipped with 3D laser scanner based on a rotary SICK LMS 100 , c) Husky platform equipped with geodetic laser system Z+F IMAGER 5010 d) a small vertical take-off aerial system (MD4-1000 from microdrones) equipped with a Sony NEX-7

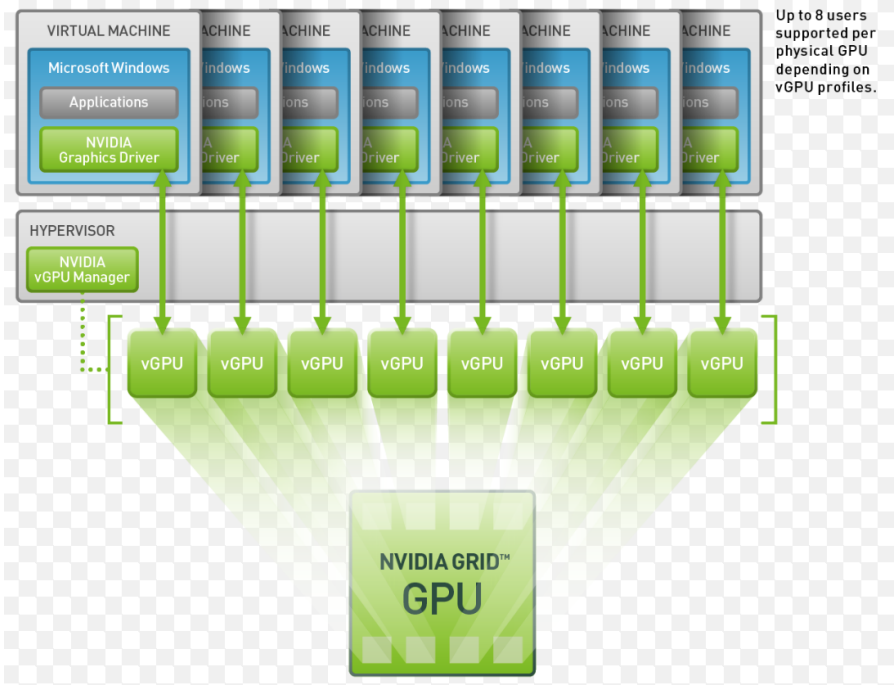

(a) Citrix VDI

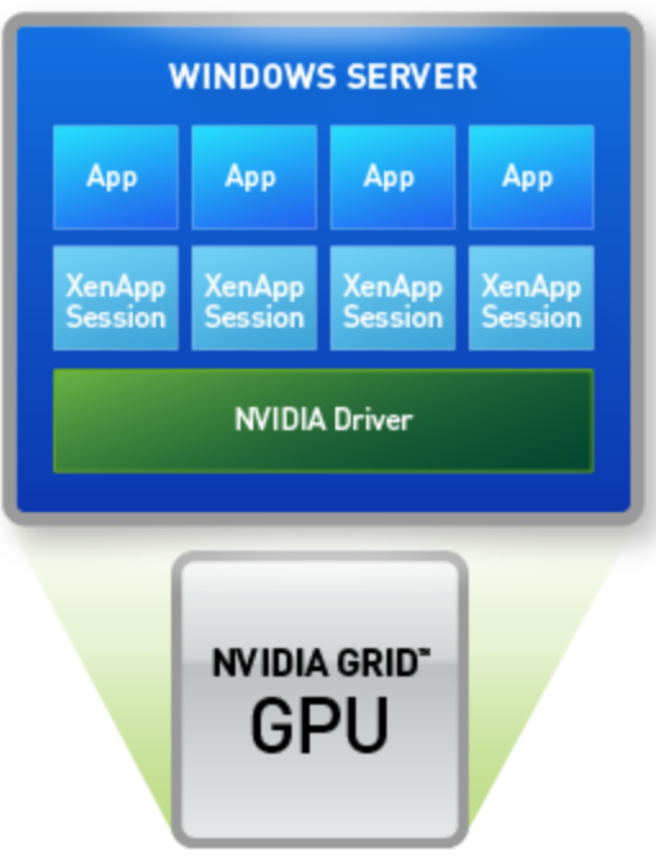

(b) Citrix XenApp

Fig. 1: Training and Support system.

high resolution digital camera with 24.3 megapixel resolution and up to 10 FPS shooting speed e) Mobile Data Center based on GRID technology. The effort needed for the deployment of the system depends on the weight and dimensions of the hardware. A minimum effort is required for the drrobot Jaguar $4 \times 4$ (one man effort) and the UAV system. The robot husky and the mobile data center are designed for two-man teams. The robot Teodor is too heavy to be carried by manpower alone, so it has to be transported with special equipment. The mobility offered by this particular platform allows performing tasks in extremely difficult conditions, where other robots would not be sufficient. 


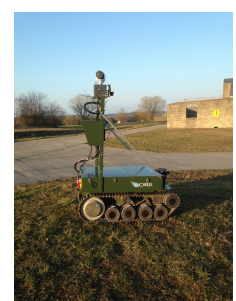

(a) Teodor

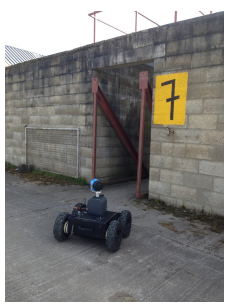

(b) Drrobot Jaguar 4x4

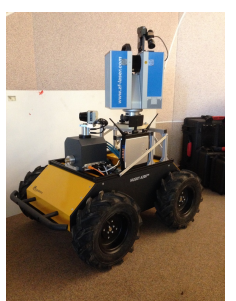

(c) Husky

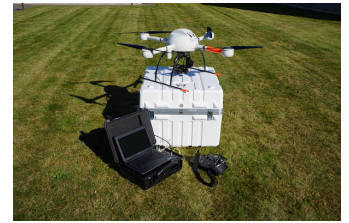

(d) Aerial system md4-1000

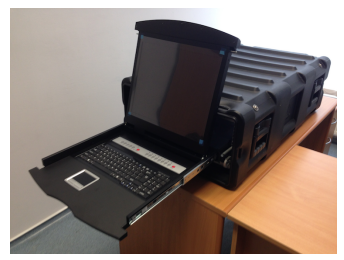

(e) Mobile Data Centre
Fig. 2: ICARUS SAR 3D mapping Hardware.

\section{Data Fusion Methodology}

This section describes the SLAM algorithm used by the 3D mapping system to fuse 3D data from all robotic sources. Figure 3 shows the scheme of the algorithm. Green rectangles correspond to software components using high performance computing with CUDA: filtering and subsampling, semantic classification and semantic 3D scan matching. Semantic approach efficiently decreases the time of the 6DSLAM convergence, by requiring lower number of iterations, especially in the situation where odometry readings are not sufficiently accurate. We consider this functionality as advantageous in extreme 3D mapping applications. It is also more reliable than state of the art approaches for demanding scenarios such as moving down stairs or mapping harsh environments. In the following sections, the main components of the algorithm will be described.

\section{A. Filtering and subsampling}

This software component prepares 3D data for further analysis. For efficient NNS (Nearest Neighbourhood Search), a regular grid decomposition (RGD) strategy is used. RGD was briefly discussed in our previous work (Bedkowski et al., 2012). The implementation allows to perform calculations for each 3D point in parallel. RGD decomposes 3D space into 64, 128, 256 or 512 buckets for each direction. For each query point, 27 surrounding buckets are searched to find the nearest neighbour. Filtering and subsampling procedures are based on NNS. For filtering, each query point with number of NN lower than a given threshold is erased from the data set. For subsampling, from each bucket only a single point, closest to the centroid of this bucket remains. It is possible to adjust the final density by choosing proper number of buckets.

\section{B. Semantic classification}

Semantic classification assigns a class (wall, ceiling, floor, edge label to each query point. This procedure is executed in parallel. The first step of the classification is normal vector estimation based on the SVD/PCA method described in our

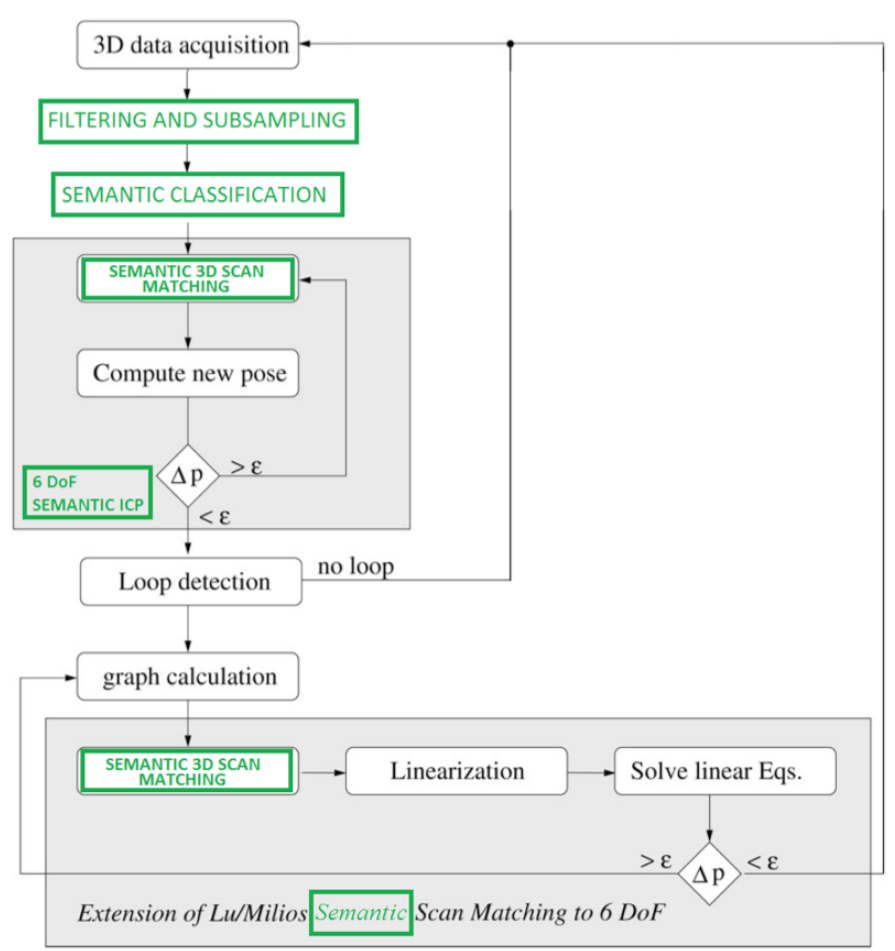

Fig. 3: Scheme of 6DSLAM algorithm.

previous work devoted to General Purpose Computing on Graphics Processing Units (GPGPU) for robotic applications (Bedkowski et al., 2013). Once normal vectors are computed for all query points, the classification procedure distinguishes points that lay on plane from those laying on edge assuming given radius (for example $25 \mathrm{~cm}$ ). For points gathered by the UGVs two additional classes can be assigned: floor, ceiling. The horizontal plane above robot is considered as the ceiling. The horizontal plane below robot is considered as the floor. The classification procedure helps in points' discrimination in the subsequent ICP (Iterative Closest Point) procedure and NNS in loop closing. It is important to emphasize that the classification accuracy is less important than classification (from the matching point of view it is more important for the same point on two scans to get the same label than the label itself). Semantic maps could be useful for End Users because adding colours decrease the cognitive load during 3D point cloud analysis and understanding.

\section{Semantic scan matching}

We improved the ICP algorithm by discriminating points into four classes during nearest neighbourhood search procedure. The key concept of the ICP algorithm can be summarized in two steps (Segal et al., 2009):

1) Compute correspondences between the two scans (Nearest Neighbour Search).

2) Compute a transformation which minimizes the distance between corresponding points.

Iteratively repeating these two steps results in convergence to the desired transformation. Semantic discrimination of these 
correspondences improves the convergence. Range images (scans) are defined as model set $M$ where

$$
|M|=N_{m}
$$

and data set $D$ where

$$
|D|=N_{d}
$$

The goal of semantic ICP is to minimize the following cost function:

$$
E(\mathbf{R}, \mathbf{t})=\sum_{i=1}^{N_{m}} \sum_{j=1}^{N_{d}} w_{i j}\left\|\mathbf{m}_{i}^{c}-\left(\mathbf{R d}_{j}^{c}+\mathbf{t}\right)\right\|^{2}
$$

where $w_{i j}$ is assigned 1 if the $i^{\text {th }}$ point of $M$ corresponds to the $j^{t h}$ point in $D$ in the sense of minimum distance and the same class c. Otherwise $w_{i j}=0$. Class $c$ discriminates points into wall, ceiling, floor or edge. $\mathbf{R}$ is the rotation matrix, $\mathbf{t}$ is the translation matrix, $\mathbf{m}_{i}^{c}$ corresponds to point $i$ of class $c$ from the model set $M, \mathbf{d}_{j}^{c}$ corresponds to point $j$ of class $c$ from the data set $D$.

\section{6DSLAM}

The core of our 6DSLAM contribution was inspired by work (Borrmann et al., 2008). To improve the accuracy and reliability of the scan matching, we added semantic discrimination of points, what was also done in (Nüchter, 2005) and (Pfaff et al., 2007). The main difference of our approach is the new semantic classification and usage of the parallel programming model for improving the performance of the computation. Another advantage of the approach is that all the needed computation can be performed in the cloud, capable of virtualizing GPU (NVIDIA GRID technology). The cloud system allows performing 3D mapping based on many sources (mobile robots equipped with 3D lasers) in parallel and then to merge all maps into a common coordinate system. In this paper we will demonstrate the 3D map building done by our cloud system, based on data from two independent UGVs and one UAV. The 3D map can be distributed in the cloud in a SaaS (Software as a Service) model, therefore many end users can have immediate access to the results of the robots' surveying.

\section{E. Discussion on the proposed approach and advantages of the semantic approach in 6DSLAM}

Figures 4 and 5 demonstrate the advantage of the semantic approach (semantic 6DSLAM) over state of the art (SoA) 6DSLAM in two different locations. In location 1 we used a 3D laser system with LMSTIM551 (271 data points per profile) and in location 2 we used LMS100 (541 data points per profile) to obtain different density of points. The influence of number of points on the computation time was discussed in detail in (Bedkowski et al., 2013). We observed that after semantic ICP, we need only 10 loop closing iterations instead of 100 iterations in SoA 6DSLAM (semantic ICP gives a better starting point for loop closing compared to SoA ICP) to have similar final 3D metric map. For this reason, we claim that the semantic approach not only guarantees a higher rate of convergence (map from Figure $4 \mathrm{~d}$ is more accurate than 4b) in the ICP phase, but also decreases computational time (less loop closing iterations Figure $4 \mathrm{e}$ and $4 \mathrm{c}$ are similar) even taking into account that classification and data discrimination procedures require more computation effort (Figure 5). Another interesting observation from Figure 4, is that the semantic approach efficiently eliminates false matching results from the floor to the ceiling already during the ICP phase. We observed that this problem was not discussed in literature.
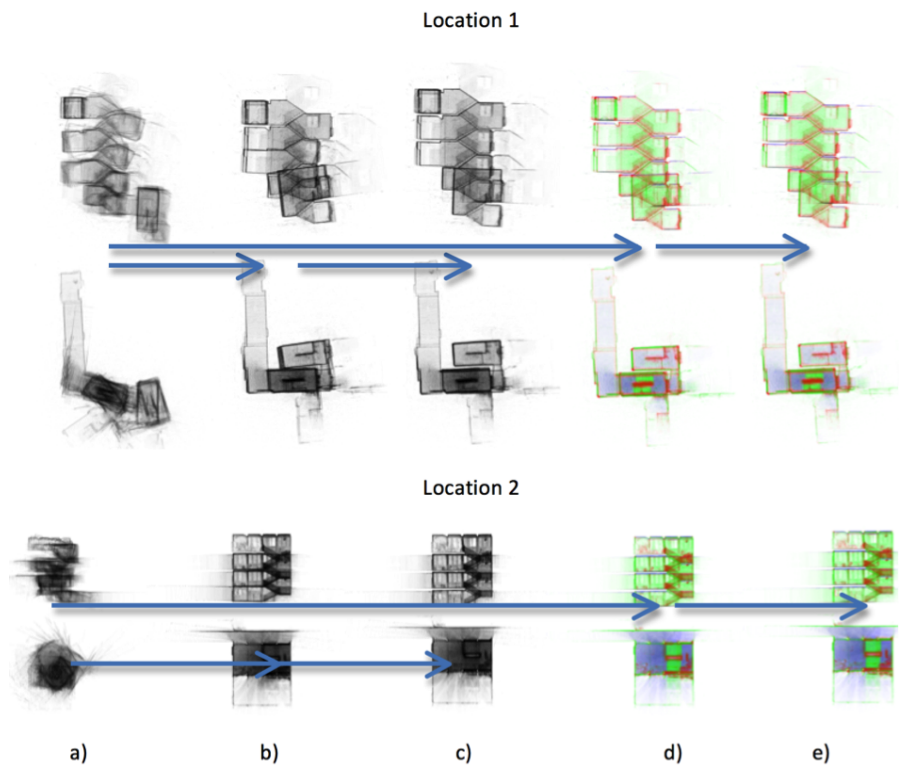

Fig. 4: Comparison of 6DSLAM with semantic 6DSLAM (row 1 and 3 are side-view, 2 and 4 are top-view); The blue arrows show the steps of semantic approach (arrows in row 1 and 3 ) and classical approach (arrows in row 2 and 4); The steps are: a) $3 \mathrm{D}$ cloud of points transformed using odometry data, b) ICP 100 iterations, c) loop closing 100 iterations, d) semantic ICP 100 iterations, e) semantic loop closing 10 iterations. It can be observed that semantic approach efficiently eliminates problem with matching of floor to ceiling already in ICP phase.
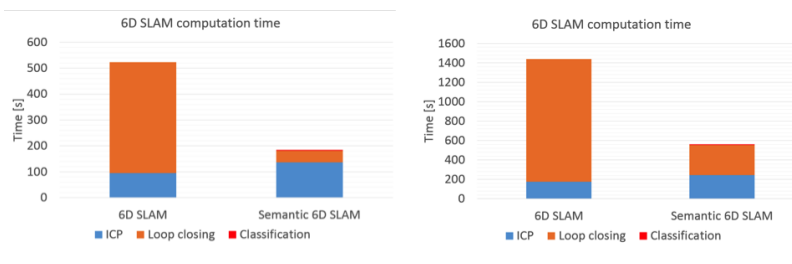

Fig. 5: Comparison of computational complexity between 6DSLAM (100 iterations of loop closing) and semantic 6DSLAM (10 iterations of loop closing). a) Left - location 1 (50 observations of $25,0003 \mathrm{D}$ data points in average). b) Right - location 2 (52 observations of 50,000 3D data points in average). Hardware GPGPU GF TITAN.

\section{EXPERIMENTS - 3D MAPPING BY UAV, 2 UGVS AND NVIDIA GRID}

In this experiment we tested merging data from three independent robotic systems. The UAV was used for image 
data collection and digital terrain model (DTM) generation. To obtain the 3D point clouds commercial Orbit UAS Mapping Software was used. The robot Jaguar $4 \times 4$ was used for collecting indoor 3D data including indoor exploration of 3 buildings. The robot Teodor was used for mapping the outdoor area.The idea is that operators have to collect the data from these robots and then transfer it to mobile data center. Once all data is in storage in the Cloud, a fourth operator performs the 6DSLAM for individual 3D cloud of points and then matches these maps using the ICP procedure. Depending on the conditions and hardware status the initial positioning of the clouds required for ICP procedure may be done automatically (GPS or good odometry and IMU readings). In case of hardware malfunction or very hard conditions (no GPS coverage, terrain to hard for proper odometry and IMU readings) the operator should perform the positioning manually. The result is shown on figure 6 , with from the left: 3D map from UAV, 3D map from drrobot Jaguar 4x4, 3D map Teodor, fused 3D map are shown. The advantage of using the mobile data center is that the 6DSLAM algorithm can be done in the cloud. Another important functionality of the system is that all renders shown in figure 6 are available in a SaaS model via XenApp for all users involved in SAR mission. The conclusion of this experiment is that the UAV data are very useful for SAR operations. The 3D laser system from the mobile ground robot can bring additional local information for extending the global operational picture. By combining aerial and ground mapping, we obtained a very satisfactory solution for SAR mapping. A better result by means of 3D cloud of points accuracy would be achieved by using a geodetic laser scanner mounted onto a UGV. For this purpose we tested our software for building 3D maps in an USAR-like location, using mentioned geodetic scanner (figure 7). Our system is able to visualise these maps as a SaaS for many End-Users over the Ethernet, therefore this could improve situational awareness of a SAR mission.
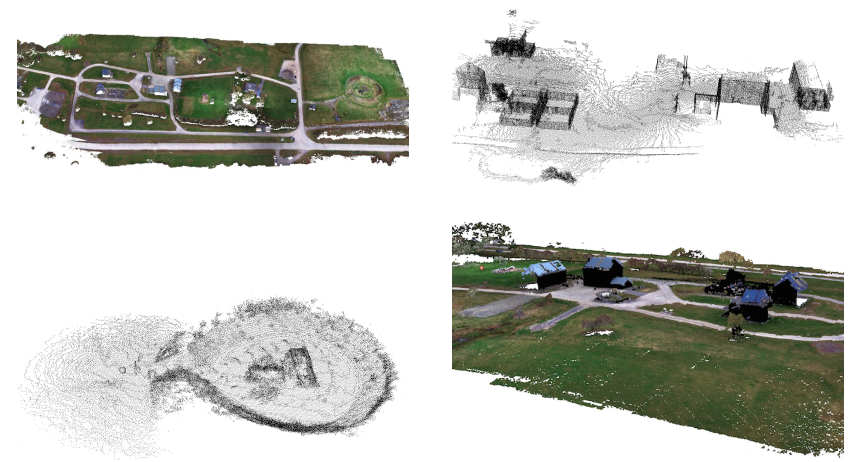

Fig. 6: Data fusion from 3D mapping systems. From left: 3D map from UAV, 3D map from drrobot Jaguar 4x4, 3D map Teodor, fused 3D map.

\section{TRAINING TOOLS}

The main goal of the training process is to familiarize the users with the ICARUS mobile platforms as well as control tools for them. To achieve this goal we designed training simulators that allow to work with simulated robots using the real controls. This is possible because simulated platforms models were developed with the same communication channels

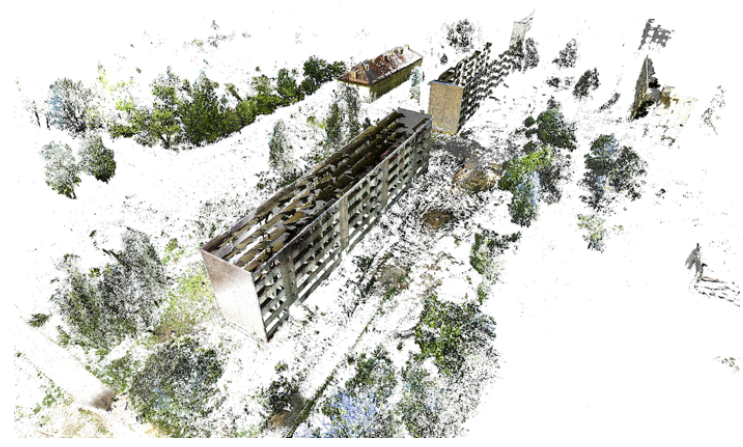

Fig. 7: 3D map obtained with geodetic terrestrial laser scanner Z+F IMAGER 5010 and ICARUS Support System.

as their real counterparts (ROS topics passed through JAUS). This approach allows to use the C2I dedicated for the real robots and provides feed of simulated sensor data such as georeference positions, cameras etc. (figure 10).

Because of major differences between areas of system's future use two simulators were developed up to this point: a ground USAR simulator and one for MSAR operations. The ground simulator is based on Vortex physics engine to achieve high fidelity of the virtual environment and interactions. The urban environment has been generated from real data gather by the platforms (figure 8). For the sea scenario, as by premise it will take place in water, a highly changeable environment, such approach was unnecessary. The sea simulator is based on Havoc engine. Both scenarios allow for multiple robots to work together on the scene (figures 9, 10).

The simulators are deployed on the GRID server using SaaS model. Apart from the connection to the real robot controls a set of virtual controls(cameras, virtual joystick) are available via XenApp client or VDI (Virtual Desktop Infrastructure). This allows the operators to conduct training from any location connected with the Data Center via internet.

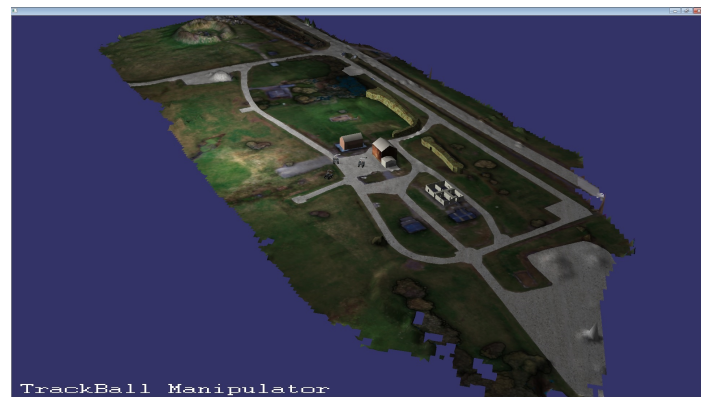

Fig. 8: Virtual environment built from data in figure 6 .

\section{CONCLUSION}

In this paper, we have presented the Training and Support system developed in the ICARUS project. The system goal is to increase the operational awareness by gathering data from the crisis area and providing it to rescuers as 3D maps. The use of a SaaS software model running from a mobile data center allows for rapid distribution of the information. Gathered data can 


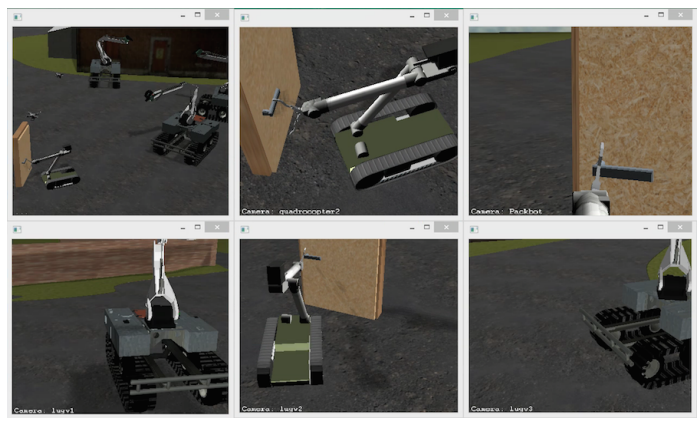

Fig. 9: Training system in the cloud - view from robots' cameras. Top middle - camera view of UAV, Top right - camera view of Small UGV, Bottom camera views of Large UGVs. The training task is opening door with Small UGV, basic control of Large UGVs and observation with UAVs.

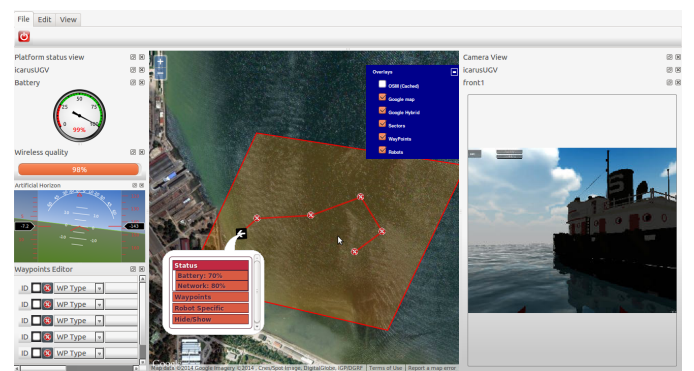

Fig. 10: MSAR mission training simulator - the C2I view of for the simulated robot

also be used for creating virtual environments that provide for better training experience. Use of GRID and HPC technologies allows for the system to work in an on-line fashion. The proposed system has been tested in realistic environment and validated by end-users. It is important to emphasize that parts of the system (the aerial system as shown on figure $2 \mathrm{~d}$ and the 3D mapping capabilities) were used during an actual relief mission in response to the 2014 floods in the Balkans (De Cubber et al., 2014). An example of gathered aerial maps is shown in figure 11 . The maps were considered highly useful for damage assessment, planning of water pumps placement and determining the natural flow of water.

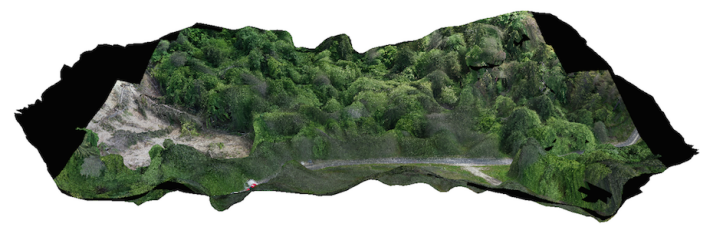

Fig. 11: Example of digital terrain model created based on data gathered during Balkan floods.

\section{ACKNOWLEDGMENT}

The research leading to these results has received funding from the European Community's Seventh Framework Programme (FP7/2007-2013) under grant agreement n285417 project ICARUS (Integrated Components for Assisted Rescue and Unmanned Search operations) and support of NCBiR (Polish Centre for Research and Development) project: Research of Mobile Spatial Assistance System Nr: LIDER/036/659/L$4 / 12 /$ NCBR/2013. The authors would like to thank Polish National Center of Science NCN for the support of project Methodology of semantic models building based on mobile robots observations Nr: DEC- 2011/03/D/ST6/03175.

\section{REFERENCES}

Bedkowski, J., Majek, K., and Nüchter, A. (2013). General Purpose Computing on Graphics Processing Units for Robotic Applications. Journal of Software Engineering for Robotics (JOSER), 4(1):23-33.

Bedkowski, J., Maslowski, A., and de Cubber, G. (2012). Real time 3D localization and mapping for USAR robotic application. Industrial Robot, 39(5):464-474.

Borrmann, D., Elseberg, J., Lingemann, K., Nüchter, A., and Hertzberg, J. (2008). Globally consistent 3d mapping with scan matching. Robot. Auton. Syst., 56(2):130-142.

De Cubber, G., Balta, H., Doroftei, D., and Baudoin, Y. (2014). Uas deployment and data processing during the balkans flooding. In 12th IEEE International Symposium on Safety, Security, and Rescue Robotics.

Dias, P. S. e. a. (2006). Mission planning and specification in the neptus framework. In ICRA, pages 3220-3225. IEEE.

Doroftei, D., De Cubber, G., and Chintanami, K. (2012). Towards collaborative human and robotic rescue workers. In Human Friendly Robotics, .

Govindaraj, S., Chintamani, K., Gancet, J., Letier, P., van Lierde, B., Nevatia, Y., De Cubber, G., Serrano, D., Esbri Palomares, M., Bedkowski, J., Armbrust, C., Sanchez, J., Coelho, A., and Orbe, I. (2013). The icarus project - command, control and intelligence (c2i). In Safety, Security, and Rescue Robotics (SSRR), 2013 IEEE International Symposium on.

Hamp, Q. e. a. (2013). Study of efficiency of usar operations with assistive technologies. Advanced Robotics, 27(5):337-350.

Kruijff, G.-J., Colas, F., Svoboda, T., van Diggelen, J., Balmer, P., Pirri, F., and Worst, R. (2012). Designing intelligent robots for human-robot teaming in urban search and rescue. In AAAI 2012 Spring Symposium on Designing Intelligent Robots. AAAI.

Liu, Y. and Nejat, G. (2013). Robotic urban search and rescue: A survey from the control perspective. Journal of Intelligent \& Robotic Systems, 72(2):147-165.

Murphy, R. (2004). Trial by fire [rescue robots]. Robotics Automation Magazine, IEEE, 11(3):50-61.

Murphy, R. R. e. a. (2008). Search and rescue robotics. In Siciliano, B. and Khatib, O., editors, Springer Handbook of Robotics, pages 1151-1173. Springer.

Nüchter, A. e. a. (2005). 3d mapping with semantic knowledge. In IN ROBOCUP INTERNATIONAL SYMPOSIUM, pages 335-346.

Pfaff, P., Triebel, R., and Burgard, W. (2007). An efficient extension to elevation maps for outdoor terrain mapping and loop closing. Int. J. Rob. Res., 26(2):217-230.

Segal, A., Haehnel, D., and Thrun, S. (2009). GeneralizedICP. In Proceedings of Robotics: Science and Systems, pages $1-8$, Seattle, USA. 